\title{
An Analysis of Curvature Based Ridge and Valley Detection
}

\author{
Siva Chandra, Jayanthi Sivaswamy \\ International Institute of Information Technology \\ Hyderabad, India \\ email: sivachandra_br@students.iiit.ac.in, jsivaswamy@iiit.ac.in
}

\begin{abstract}
A 2D function, representing a digital image, is a surface in 3D space. Curvature of such a surface can be exploited to detect ridge and valley like features from images. In this paper, we present an analysis of such curvature based ridge and valley detection techniques and come up with a description for the different classes of ridge and valley profiles which can be detected by them. Such an analysis helps in understanding the scope and limitations of the curvature based techniques. As curvature is a measure of 'bend' in the cross-section profile along a particular direction of the image intensities, the analysis is presented using 1D functions which represent cross-section profiles of ridges and valleys. The classes of profiles which can be detected by a curvature based technique are described in terms of the properties of the second-derivative of the $1 \mathrm{D}$ profile function.
\end{abstract}

\section{INTRODUCTION}

Intensity ridges and valleys form medial structures of objects in $2 \mathrm{D}$ digital images [1] such as blood-vessels in medical images and roadnetworks in satellite images. The notion of a ridge or a valley, in a 2D digital image, is formed by visualising the image function as a surface in $3 \mathrm{D}$ space. Geometric properties of such a surface can be exploited to detect image features. One such property, the curvature of the 'image surface', has been used to detect intensity ridges and valleys from 2D digital images [1] [5] [4] [3]. Surface curvature is a measure of the bend in the image surface along a particular direction. Medial lines of ridges and valleys are characterised by high magnitudes of curvature along the direction perpendicular to the orientation of the ridge.

An image feature can occur in various forms. For example, ridges and valleys can occur with different cross-sectional profiles. The scope of a feature detection technique can be determined by the different forms of a particular feature that are detected by it. By the same token, its limitations can be determined by the forms of the feature which are not detected by it. It is of importance to know the scope of a feature detection technique in order to determine its appropriateness for a given application. Evaluations of different ridge detection techniques, with respect to a list of desirable properties, and their suitability for a specific application are presented in [3] and [4]. However, in both [3] and [4], the scope and limitations of ridge detection techniques are illustrated using few specific examples. To our knowledge, there is no work in literature which tries to exhaustively establish the scope of curvature based ridge and valley detection techniques using a theoretical analysis. In this paper, we present an analysis of the general framework of curvature based ridge/valley detection to establish the scope and limitations of the techniques built on this framework. The scope of the framework is presented in terms of the different classes of ridge/valley profiles which can be detected by it.

In the next section (Section 2) we present a brief overview of the general framework of curvature based ridge and valley detection. In Section 3, we present a detailed analysis of this framework, using $1 \mathrm{D}$ cross-section profiles of ridges and valleys, and summarise the various classes of ridge and valley profiles which can be detected. In Section 4, we conclude by briefly mentioning the limitations of the framework.

\section{RIDGE AND VALLEY DETECTION FROM CURVATURE OF THE IMAGE SURFACE}

The general framework for curvature based ridge and valley detection is based on the differential geometry of image surfaces [5] [1]. For every point on a surface, there exist two curvature measures, namely, the maximum and minimum principal curvatures along two orthogonal principal directions. These measure the maximum and minimum bend in the surface. A pixel is defined as a ridge or a valley pixel if the magnitude of the maximum principal curvature (MPC) at that pixel is a local maximum in some direction and has a value greater than a chosen threshold. Depending on the reference coordinate system, a high negative curvature indicates a strong ridge strength while a high positive curvature indicates a strong valley strength, or vice versa. The direction along which the MPC is a maximum is the direction perpendicular to the orientation of the ridge (or valley) at that pixel. An efficient computational algorithm for such a curvature based detection framework can be found in [5]. Here, the ridge and valley pixels are located at the zero crossings of the directional derivative of the magnitude of the MPC along the corresponding principle directions. Zerocrossings of the directional derivative would also correspond to minima of the magnitude of MPC. However, such minima are rejected by a thresholding operation.

\section{ANALYSIS}

As mentioned in the previous section, the framework for curvature based detection of ridges and valleys is based on differential geometry of 3D surfaces (Section 2). However, curvature of an image function is a measure of the 'bend' in the cross-section profile along a particular direction of the image intensity values. Hence, analysis of 
curvature based feature detection technique can be done using just 1D functions which represent the (cross-section) profiles of the features of interest. In this section, we present an analysis of the curvature based ridge/valley detection framework (Section 2) using 1D profiles of ridges and valleys.

A ridge/valley detection technique detects medial lines of such structures. Such medial lines are loci of 'medial points' of the cross-section profiles of ridges/valleys. Therefore, in order to use 1D profile functions to perform an analysis of curvature based ridge/valley detection, the original 2D framework has to be reformulated to detect medial points of 1D profile functions. Before presenting such a reformulation, we shall define a few terms for ease of presentation later in this paper and state a Lemma.

Definition 1 (Point of Magnitude Maximum): Let $f: \Re \rightarrow \Re$ be a 1D function. If a point $x=a$ is a point of local maximum of the function $y=|f(x)|$, then it is a point of magnitude maximum of the function $y=f(x)$. For brevity, we shall refer to such a point of magnitude maximum of a function as a PMMAX of the function.

Definition 2 (Point of Magnitude Minimum): Let $f: \Re \rightarrow \Re$ be a $1 \mathrm{D}$ function. If a point $x=a$ is a point of local minimum of the function $y=|f(x)|$, then it is a point of magnitude minimum of the function $y=f(x)$. For brevity, we shall refer to such a point of magnitude minimum of a function as a PMMIN of the function.

Lemma 1: Let $f: \Re \rightarrow \Re$ be a 1D function for which derivatives upto the second order exist. If

$$
\text { (a) }\left[\frac{d y}{d x}\right]_{x=a}=0 \text { and, (b) }\left[y \frac{d^{2} y}{d x^{2}}\right]_{x=a}<0
$$

then, $x=a$ is a PMMAX of the function $y=f(x)$.

\section{Proof: See APPENDIX A}

The curvature of a $1 \mathrm{D}$ function $y=f(x)$ is given as [2]:

$$
\kappa(x)=\frac{\frac{d^{2} y}{d x^{2}}}{\left\{1+\left(\frac{d y}{d x}\right)^{2}\right\}^{\frac{3}{2}}}
$$

We shall now state the criterion for curvature based medial point detection using Definition 1.

Definition 3 (Curvature based criterion for medial points of 1D profile functions): Let $f: \Re \rightarrow \Re$ be a $1 \mathrm{D}$ function for which derivatives upto the second order exist. A point $x=a$ is a medial point of the profile function $y=f(x)$ if it is a PMMAX of $\kappa(x)$.

[ NOTE: Later in this paper, we will require that $\kappa(x)$ be twice differentiable. This in turn would require that $y=f(x)$ has derivatives upto the fourth order. ]

The PMMAX of the curvature is where the derivative of the curvature vanishes. The derivative of the curvature is found, by differentiating the expression in equation 3.1:

$$
\frac{d \kappa}{d x}=\frac{\frac{d^{3} y}{d x^{3}}\left\{1+\left(\frac{d y}{d x}\right)^{2}\right\}-3 \frac{d y}{d x}\left(\frac{d^{2} y}{d x^{2}}\right)^{2}}{\left\{1+\left(\frac{d y}{d x}\right)^{2}\right\}^{\frac{5}{2}}}
$$

Considering equation 3.2, it is clear that the first derivative of the curvature can vanish under four different conditions. These are:

$$
\begin{aligned}
& \text { C1 }:: \quad \frac{d y}{d x}=0, \frac{d^{2} y}{d x^{2}} \neq 0, \frac{d^{3} y}{d x^{3}}=0 \\
& \mathrm{C} 2:: \quad \frac{d y}{d x}=0, \frac{d^{2} y}{d x^{2}}=0, \frac{d^{3} y}{d x^{3}}=0 \\
& \mathrm{C} 3 \quad:: \quad \frac{d y}{d x} \neq 0, \frac{d^{2} y}{d x^{2}}=0, \frac{d^{3} y}{d x^{3}}=0 \\
& \mathrm{C} 4 \quad:: \quad \frac{d y}{d x} \neq 0, \frac{d^{2} y}{d x^{2}} \neq 0, \frac{d^{3} y}{d x^{3}} \neq 0 \text { but the numerator as a } \\
& \text { whole, of the expression on the right hand side } \\
& \text { of equation } 3.2, \text { goes to zero. }
\end{aligned}
$$

The second derivative of the profile function is zero in C2 and C3. Hence, by equation 3.1, the curvature of the profile function also goes to zero at such points. Therefore, a point satisfying $\mathrm{C} 2$ or $\mathrm{C} 3$ cannot be a PMMAX of the curvature function. A medial point is either the top of a ridge profile, or the bottom of a valley profile. In other words, the medial points are points of extremal image intensities. Hence, a PMMAX which satisfies $\mathrm{C} 4$ cannot be a medial point of a ridge/valley profile. Such PMMAX occur as 'knee/elbow' points of edge profiles, as shown in Figure 1. In practice, it is either rejected by setting a threshold or in few rare cases, is wrongly classified as a ridge/valley pixel. Therefore, medial points which satisfy the criterion in Definition 3 should satisfy only $\mathrm{C} 1$. However, a point satisfying $\mathrm{C} 1$ need not satisfy the criterion in Definition 3. The following theorem gives us a condition under which a point satisfying $\mathrm{C} 1$ is also a PMMAX of the curvature function.

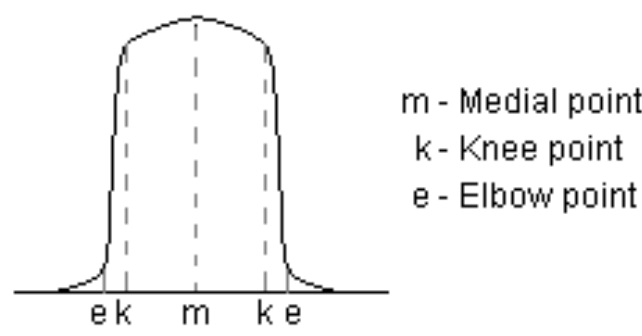

Figure 1. Cross-section of a ridge and the various points of extremal curvature.

Theorem 1: Let $f: \Re \rightarrow \Re$ be a $1 \mathrm{D}$ function for which derivatives upto the fourth order exist. If for some point $x=a$, we have $\left[\frac{d y}{d x}\right]_{x=a}=0,\left[\frac{d^{2} y}{d x^{2}}\right]_{x=a} \neq 0,\left[\frac{d^{3} y}{d x^{3}}\right]_{x=a}=0$, and 


$$
\left[\frac{d^{2} y}{d x^{2}}\left\{\frac{d^{4} y}{d x^{4}}-3\left(\frac{d^{2} y}{d x^{2}}\right)^{3}\right\}\right]_{x=a}<0
$$

then, $x=a$ is a PMMAX of the curvature of function $y=f(x)$.

Proof: Follows trivially by applying Lemma 1 to the curvature expression in equation 3.1

If at some point on the profile, the fourth-derivative is non-zero; the curvature function has a PMMAX; and $\mathrm{C} 1$ is satisfied; then, the profile function has to satisfy an inequality. This is stated in the following theorem.

Theorem 2: Let $f: \mathfrak{R} \rightarrow \mathfrak{R}$ be a $1 \mathrm{D}$ function for which derivatives upto fourth order exist. If some point $x=a$ is a PMMAX of the curvature of the function $y=f(x)$ while satisfying $\left[\frac{d y}{d x}\right]_{x=a}=0$,

$$
\begin{aligned}
{\left[\frac{d^{2} y}{d x^{2}}\right]_{x=a} \neq 0, } & {\left[\frac{d^{3} y}{d x^{3}}\right]_{x=a}=0 \text { and }\left[\frac{d^{4} y}{d x^{4}}\right]_{x=a} \neq 0, \text { then } } \\
& {\left[\frac{d^{2} y}{d x^{2}}\left\{\frac{d^{4} y}{d x^{4}}-3\left(\frac{d^{2} y}{d x^{2}}\right)^{3}\right\}\right]_{x=a}<0 }
\end{aligned}
$$

\section{Proof: See APPENDIX A}

$\mathrm{C} 1$ requires the third-derivative to be zero while the second-derivative is non-zero. Let $x=a$ be a point where $\mathrm{C} 1$ occurs. Then, we will have the following possible properties for the second-derivative function at that point

\section{A. $x=a$ is a PMMAX of the second-derivative function:}

Since the denominator of the curvature expression (equation 3.1) is always positive, the point $x=a$ is a PMMIN of the denominator of the curvature expression. Furthermore, given that the point $x=a$ is a PMMAX of the second-derivative implies that it is also a PMMAX of the numerator of the curvature expression. Therefore, we can conclude that $x=a$ is a PMMAX of the curvature expression as a whole.

B. $x=a$ is a point of inflection of the second-derivative function which is non-zero at this point:

$$
\begin{aligned}
& \text { In this case, we must have }\left[\frac{d^{4} y}{d x^{4}}\right]_{x=a}=0 \text {. Hence, we have } \\
& \qquad\left[\frac{d^{2} y}{d x^{2}}\left\{\frac{d^{4} y}{d x^{4}}-3\left(\frac{d^{2} y}{d x^{2}}\right)^{3}\right\}\right]_{x=a} \\
& =\left[\left\{-3\left(\frac{d^{2} y}{d x^{2}}\right)^{4}\right\}\right]_{x=a}<0
\end{aligned}
$$

Therefore, by Theorem 1, the point $x=a$ is also a PMMAX of the curvature function. An example of a profile function with such a PMMAX is $f(x)=x^{5}+10 x^{2}, x \in[-1,1]$ and its second derivative is $f^{\prime \prime}(x)=20 x^{3}$. $x=0$ is a point of inflection of the second-derivative, which is also a PMMAX of its curvature function.

C. The second-derivative is a non-zero constant function:

In this case again, the fourth derivative must vanish, i.e., $\left[\frac{d^{4} y}{d x^{4}}\right]_{x=a}=0$. Hence, as in case B above, the point $x=a$ is a PMMAX of the curvature function. Examples of such profiles are quadratic polynomials which have a unique point of minimum or maximum.

D. $x=a$ is a PMMIN of the second-derivative function which is non-zero here; the fourth derivative vanishes at this point:

Using Theorem 1 again, as with cases $\mathrm{B}$ and $\mathrm{C}$ above, it can be concluded that $x=a$ is a PMMAX of the curvature function.

E. $x=a$ is a PMMIN of the second-derivative function and the fourth and the second derivatives are non-zero at this point:

Using Theorems 1 and 2, $x=a$ is a PMMAX of the curvature function only if the derivatives of the profile function satisfy the inequality 3.4 .

The five different cases discussed above correspond to five classes of profiles for which a point satisfying $\mathrm{C} 1$ is also a PMMAX of the curvature function. Hence, we conclude that there are five different classes of ridge and valley profiles which can be detected by techniques using the curvature based criterion in Definition 3. These classes are summarised below for easy reference, in terms of the characteristics of the function at the medial point.

Class 1 Functions for which there exists a PMMAX of the secondderivative at a point where the first derivative vanishes.

Class 2 Functions for which there exists a point of inflection of the second-derivative at a point where the first-derivative vanishes and the second-derivative has a non-zero value.

Class 3 Functions for which the second-derivative is a non-zero constant function and there exists a point where the first derivative vanishes.

Class 4 Functions for which there exists a PMMIN of the secondderivative at a point where the first and fourth derivatives vanish, and the second-derivative has a non-zero value.

Class 5 Functions for which there exists a PMMIN of the secondderivative at a point where the fourth-derivative is nonzero, and condition $\mathrm{C} 1$ and the inequality 3.4 are satisfied at that point.

\section{CONCLUSION}

An analysis to determine the scope of curvature based ridge and valley detection techniques was presented in Section 3. It is important to know the scope of a feature detection technique in order to determine its suitability for a specific application. Since curvature is a directional measure, it is more convenient to use 1D (cross-sectional) profiles of the features of interest to perform an analysis of curvature based feature detection techniques. Such an approach was used in Section 
3, and it was shown that curvature based tecchniques can detect five different classes of ridge and valley profiles. This approach based on 1D profile functions, can also be extended to perform a scale-space analysis of curvature based ridge/valley detection techniques.

Curvature based ridge/valley detection techniques reject two kinds of points: non-extrema and PMMINs of the curvature function. Nonextremum value, at some point of the curvature function, implies existence of points with higher curvature (magnitude) in the neighbourhood of such a point. Hence, rejection of such points does not lead to omission of ridge/valleys pixels. On the other hand, rejection of PMMINs of the curvature function will lead to omission of few ridge/valley pixels. Examples are valleys with profile functions of type $y=x^{2 n},|x| \leq 1$, where $n$ is a positive integer greater than 1 . The medial point of such valley profiles is at $x=0$, where the secondderivative of the profile function vanishes making the curvature zero (see equation 3.1). Such a medial point is a PMMIN of the profile function and is rejected by curvature based techniques.

\section{APPENDIX A. PROOFS}

\section{Proof of Lemma 1}

$$
\begin{aligned}
& f^{\prime}(a)=0, f(a) f^{\prime \prime}(a)<0 \\
\Rightarrow & f(a)<0, f^{\prime}(a)=0, f^{\prime \prime}(a)>0 \\
& \text { or } \\
& f(a)>0, f^{\prime}(a)=0, f^{\prime \prime}(a)<0 \\
\Rightarrow & f(a)<0, f(a)<f(b), b \in(a-\delta, a+\delta)-\{a\} \quad \text { for some } \\
& \delta>0 .\left(\text { Since } f^{\prime}(a)=0, f^{\prime \prime}(a)>0 \text { implies that } x=a\right. \text { is a point } \\
& \text { of minimum of } f(x)) \\
& f(a)>0, f(a)>f(b), b \in(a-\delta, a+\delta)-\{a\} \quad \text { for some } \\
& \delta>0 .\left(\text { Since } f^{\prime}(a)=0, f^{\prime \prime}(a)<0 \text { implies that } x=a\right. \text { is a point } \\
& \text { of maximum, of } f(x)) \\
\Rightarrow \quad & |f(a)|>|f(b)|, b \in(a-\delta, a+\delta)-\{a\} \text { for some } \delta>0 . \\
\Rightarrow & x=a \text { is a point of maximum of the function } y=|f(x)| .
\end{aligned}
$$

Hence, Lemma 1 is proved.

\section{Proof of Theorem 2}

The curvature function $\kappa(x)$, around the number $a$, can be evaluated using the Taylor's series as:

$$
\kappa(x)=\kappa(a)+(x-a) \kappa^{\prime}(a)+\frac{(x-a)^{2}}{2 !} \kappa^{\prime \prime}(a)+\ldots
$$

Under the conditions of the theorem, $\kappa^{\prime}(a)$ is zero as $\frac{d y}{d x}=0$ and $\frac{d^{3} y}{d x^{3}}=0$. Let a function $R(x)$ be defined as:

$$
R(x)=\frac{(x-a)^{2}}{2 !} \kappa^{\prime \prime}(a)+\frac{(x-a)^{3}}{3 !} \kappa^{\prime \prime \prime}(a)+\ldots
$$

Under the conditions of the theorem, equation A.1 can be written in terms of $R(x)$ as:

$$
\kappa(x)=\kappa(a)+R(x)
$$

Let $\kappa(a)>0$. If $x=a$ is a PMMAX of the function $\kappa(x)$, then it must follow from equation A.3 that $R(x)<0$ in the neighbourhood of $a$. Similarly, if $\kappa(a)<0$, it must follow that $R(x)>0$ in the neighbourhood of $a$. These two cases can be captured in one single inequality as:

$$
R(x) \cdot \kappa(a)<0
$$

in some neighbourhood of $a$. For a given ridge/valley profile, the derivatives $\left\{\kappa^{\prime \prime}(a), \kappa^{\prime \prime \prime}(a), \ldots\right\}$ are all constants. Moreover, the coefficient of $\kappa^{\prime \prime}(a)$ in the expression for $R(x)$ (see equation A.2) is always positive. Hence, there will exist a $\delta$ such that:

$$
R(x) \cdot \kappa^{\prime \prime}(a)>0, x \in(a-\delta, a+\delta)-\{a\}
$$

In other words, the above inequality says that by selecting an $x$ close to $a$, we can make the sign of $R(x)$ same as that of $\kappa^{\prime \prime}(a)$. This is due to the diminishing values (in magnitude) of the coefficients of the higher order terms, relative to the coefficient of $\kappa^{\prime \prime}(a)$, as $x$ gets closer to $a$. Using this equality in sign (of $\kappa^{\prime \prime}(a)$ and $R(x)$ ) in the neighbourhood of $a$, we can transform the inequality A. 4 into:

$$
\kappa^{\prime \prime}(a) \cdot \kappa(a)<0
$$

Under the conditions of the theorem, the above inequality can be expressed in terms of the derivatives of the function $y=f(x)$ as:

$$
\left[\frac{d^{2} y}{d x^{2}}\left\{\frac{d^{4} y}{d x^{4}}-3\left(\frac{d^{2} y}{d x^{2}}\right)^{3}\right\}\right]_{x=a}<0
$$

Hence, Theorem 2 is proved.

\section{REFERENCES}

[1] D. Eberly, R. Gardner, B. Morse, S. Pizer, C. Scharlach. Ridges for image analysis. Journal of Mathematical Imaging and Vision, 4:353-373 (1994).

[2] Erwin Kreyszig. Advanced Engineering Mathematics. Wiley. Eight Edition, 1998.

[3] A. Lopez, F. Lumbreras, J. Serrat and J. Villanueva. Evaluation of methods for ridge and valley detection. IEEE Trans. on PAMI, 21(4):327-335 (1999).

[4] J. B. A. Maintz, P. A. van den Elsen, M. A. Viergever. Evaluation of Ridge Seeking Operators for Multimodality Medical Image Matching. IEEE Trans. on PAMI, 18(4) (1996).

[5] O. Monga, N. Armande, P. Montesinos. Thin nets and Crest lines: Applications to Satellite Data and Medical Images. Proceeding of the IEEE Intl. Conf. of Image Procc. (1995). 